\title{
Editorial: Corona und die Folgen: Gewinner, Verlierer und Chancen für eine neue Gesellschaftspolitik
}

er Beginn der COVID-19-Pandemie
liegt inzwischen zwei Jahre zurück. In der politischen und sozialwissenschaftlichen Debatte wurde oft betont, sie wirke wie ein Brennglas und zeige, wo soziale Prioritäten liegen müssten, entlang welcher Linien Ungleichheiten die Gesellschaft strukturieren, was in ihr gut, und was nicht so gut funktioniert. Besonders deutlich wurde dies in den Gesundheitssystemen: In Anbetracht von knappen medizinischen Behandlungs- und Bettenkapazitäten sowie vielerlei Liefer- und Produktionsengpässen ist die Notwendigkeit einer funktionierenden Notfall-, Gesundheits- und Arzneiversorgung schlagartig in den Fokus von Politik, Wissenschaft und Öffentlichkeit gerückt. Zentrale Probleme des Gesundheitswesens konnten aber auch nach zwei Jahren nicht gelöst werden. So wurden in Deutschland im Jahr 2021 trotz Corona ca. 4.000 Intensivbetten in Krankenhäusern abgebaut, da es an Pflegepersonal fehlt.

Aber die Auswirkungen der Pandemie beschränken sich nicht auf das Gesundheitswesen, Schwerpunkt der PROKLA 205. Die spezifische Kombination von Einbrüchen auf der Angebots- und auf der Nachfrageseite der
Wirtschaft hat im globalen Maßstab zu Rückgängen geführt, die die Effekte früherer Krisen wie etwa der Finanzkrise 2008/2009 übersteigen. Eine Besonderheit war diesmal, dass das staatliche Handeln nicht einfach darauf abzielen konnte, mit einschlägigen Maßnahmen die Wirtschaftsbelebung anzukurbeln. Im Gegenteil brachten Lockdowns insbesondere in den konsumorientierten Dienstleistungsbereichen die Wirtschaft zeitweilig fast zum Erliegen. Zur Kompensation wurden in unterschiedlichem Umfang konjunktur- und sozialpolitische Maßnahmen ergriffen. In Deutschland etwa wurde der Zugang zum Kurzarbeitergeld erleichtert und ein CoronaKonjunkturprogramm aufgelegt. Mehr als 130 Milliarden Euro wurden eingesetzt, um die Folgen der Coronapandemie und der größten Wirtschaftskrise der Nachkriegszeit zu bewältigen. Die EU beschloss einen 750 Milliarden Euro umfassenden Wiederaufbaufonds. Und in den USA legte die Biden-Regierung ein 1,9 Billionen US-Dollar schweres Konjunkturprogramm auf.

Die Pandemie führte nicht nur zu einer Wirtschaftskrise und zu umfassenden staatlichen Interventionen, die gänzlich undenkbar schienen, solange 
das fiskalpolitische Dogma der Schuldenbremse hochgehalten wurde. Sie hat auch in aller Deutlichkeit soziale Ungleichheiten offengelegt, die innerhalb der Nationalstaaten und im globalen Maßstab sowohl den Zugang zur Gesundheitsversorgung wie die Einkommensund Vermögensverhältnisse betreffen. Die Gefahr, an Corona zu erkranken und möglicherweise schwere Krankheitsverläufe zu erleiden, ist extrem ungleich verteilt und betrifft allerorten Menschen mit geringeren Einkommen sehr viel stärker als die sozial Bessersituierten. Entscheidende Faktoren sind hier Wohn- ebenso wie Arbeitsverhältnisse. Während viele, die von vornherein einen besseren Gesundheitsstatus hatten, sich ins Homeoffice innerhalb komfortabler Wohnungen zurückziehen konnten, mussten andere in Versandlagern und an Supermarktkassen, in Krankenhäusern oder Schlachtbetrieben weiter im direkten Kontakt mit Kolleg*innen, Kund*innen oder Patient*innen arbeiten und waren Coronaausbrüchen ausgeliefert. Die krassen Ungleichheiten setzten sich beim Zugang zu Impfungen fort. In den USA etwa mussten sich Schwarze, Latinos und Latinas oder andere benachteiligte Bevölkerungsgruppen angesichts des gängigen Systems der Priorisierungen bei den Atemschutzgeräten wie bei den Impfungen weit hinten anstellen. Ähnlich sieht es auf der internationalen Ebene aus. Während in vielen Ländern des globalen Nordens mit den Boostern bereits massenhaft Drittimpfungen erfolgen, erreichen die Impfquoten in vielen Ländern in Afrika häufig nicht einmal zehn Prozent der Bevölkerung. Hierfür ist auch die fehlende Freigabe von $\mathrm{Pa}-$ tenten auf die Impfstoffe verantwortlich.
Neben den gravierenden Ungleichheiten der Gesundheitsversorgung ist nach Gewinnern und Verlierern bei Einkommen und Vermögen zu fragen. Die Unterstützungsprogramme haben in europäischen Ländern oder den USA zumindest vorübergehend krasse Formen der Not bei denen verhindert, die ihre Arbeit verloren oder sonst erhebliche Einkommensverluste erlitten. Auch Selbstständige und Unternehmen wurden massiv unterstützt, wenngleich die Kriterien, nach denen enorme Summen ausgeschüttet wurden, häufig im Dunkeln blieben. Dennoch zeigen erste Befunde, dass jene, die besonders große Vermögen besitzen, hierbei besonders profitiert haben. Gerade die Allerreichsten sind eindeutige Gewinner der Umverteilung, die im Zug der Pandemie und der damit verbundenen Maßnahmen stattgefunden hat. Die weltweit 2.700 Milliardäre und Milliardärinnen haben ihr Vermögen im Jahr 2020 um sechs Prozent steigern können.

Die Beiträge des Schwerpunkts widmen sich unterschiedlichen Facetten der Pandemie, insbesondere ihren politischen, sozialen und ökonomischen. Alex Demirović geht den Reaktionen der gesellschaftlichen Linken auf Covid-19 und der ihr oftmals vorgeworfenen Handlungsunfähigkeit nach. Er zeigt, wie sehr die Pandemie die theoretischen Koordinaten der staatstheoretischen Analyse in einem gewissen Maß irritiert, und unterzieht die bisherige Coronapolitik einer materialistischstaatstheoretischen Analyse, bei der er Erkenntnisse von Joachim Hirsch, Michel Foucault und Nicos Poulantzas diskutiert. Seine Auseinandersetzung mit dem Thema wird Demirović in einer der kommenden Ausgaben fortsetzen. 
Philipp Köncke und Stefan Schmalz beschäftigen sich angesichts der globalen Unterschiede in der Impfstoffnutzung mit Ungleichheiten und geopolitischen Konflikten in der Coronapandemie. Sie gehen davon aus, dass mehrere »Impfstoffwelten « entstanden sind. Von diesen sind die Bevölkerungen der ersten quantitativ und qualitativ relativ gut versorgt, anders diejenigen der zweiten und dritten Impfstoffwelten. Dort vollziehen sich außerdem geopolitische Konflikte, da der Wettbewerb bei der Impfstoffproduktion und-distribution zwischen Herstellerfirmen aus den USA und der EU einerseits und chinesischen Produzenten andererseits stattfindet und in seinen Frontstellungen den bereits zuvor ausgetragenen geopolitischen Auseinandersetzungen entspricht.

Lorena Herzog nimmt einen der großen Gewinner der Pandemie in den Blick, den US-amerikanischen Technologiekonzern Amazon. Sie skizziert die Markt- und Geschäftsentwicklung des Unternehmens in unterschiedlichen Feldern und zeigt, dass nicht - wie häufig angenommen wird - dessen enorme Umsätze im Versandhandel besonders profitabel waren, sondern vor allem das Geschäft des Cloud-Computings, mit dem das hohe Wachstum der Umsätze und der Gewinne erklärt werden kann.

Kathrin Schäfers, Jochen Schroth und Manfred Wannöffel setzen sich in ihrem Artikel mit der Entwicklung von grenzüberschreitenden Wertschöpfungsketten und der Kooperation von Arbeitnehmer*innen in Unternehmensnetzwerken auseinander. Am Beispiel der globalen Wertschöpfungsketten der deutschen Automobil- und Zulieferindustrie und speziell ihrer Produktions- standorte in Mexiko und Südafrika wird diskutiert, vor welchen besonderen Herausforderungen die transnationale Gewerkschaftsarbeit bei der Überwindung der Coronakrise steht, und wie die Einhaltung von globalen Arbeitsund Sozialstandards an internationalen Produktionsstandorten deutscher Unternehmen gelingen kann.

Stefanie Hürtgen greift in ihrem Beitrag die Debatte um systemrelevante Arbeit auf, die insbesondere zu Beginn der Pandemie geführt wurde. Ihr geht es dabei um eine demokratietheoretische Erweiterung dieser Debatte. Sie zeigt, warum auch politisch eingreifende Artikulationen von Arbeiter*innen als »systemrelevant « gelten können und deren Kritik an gegenwärtigen Arbeits- und Lebensbedingungen eine demokratiepolitische Auseinandersetzung darstellt, in der das gesellschaftliche Verständnis von (Lohn-)Arbeit auf dem Spiel steht.

Außerhalb des Schwerpunkts untersucht Felix Syrovatka die sozialpolitischen Initiativen der EU seit 2017. Er hinterfragt die Annahme, diese würden einen grundlegenden Paradigmenwechsel darstellen, und argumentiert, sie zeichneten sich vielmehr durch einen weitgehend symbolischen Charakter aus und resultierten aus politischen Desintegrationstendenzen sowie neuen geopolitischen Herausforderungen. Des Weiteren befasst sich Alexander Lenk mit dem Problem der akademischen Prekarität und führt aus, inwieweit diese zu einem neoliberalen Modus von Subjektivierung avanciert ist. Aus Interviews mit Wissenschaftler*innen rekonstruiert er unterschiedliche Deutungsmuster von akademischer Prekarität wie 
die Selbstaktivierung, den »heilsamen Selbstschutz«, den Balanceakt, den Einzelkampf oder die Freiheitsberaubung.

Am 30. November des letzten Jahres ist Bodo Zeuner gestorben. Wir haben diese Nachricht mit großem Bedauern aufgenommen. Bodo war der PROKLA bereits seit ihren Anfängen über viele Jahrzehnte verbunden und hat eine Reihe von Beiträgen verfasst, die bis heute ebenso relevant wie anregend sind und in unserem Archiv nachgelesen werden können (online unter prokla.de). Der erste davon entstand 1974 zusammen mit Siegfried Heimann, der für dieses Heft auch einen Nachruf verfasst hat. Der gemeinsame Artikel in Heft 14/15 war der damaligen Entwicklung der Politik der SPD und ihres sozialreformerischen Programms gewidmet, und zwar anhand der Diskussion und Kritik einer Broschüre von Peter von Oertzen, zu dieser Zeit Vorsitzender der SPD-Langzeitprogramm-Kommission. Die SPD war auch Thema in einem Beitrag in Heft 26 von 1977. Unter dem Titel "Solidarität" mit der SPD oder Solidarität der Klasse wurde diesmal ihre Bedeutung für die Gewerkschaften, die von vielen zunehmend als sozialdemokratische Richtungsgewerkschaften gesehen wurden, untersucht. Ein weiterer Beitrag aus den 1970erJahren wurde unter dem Titel Die innerwissenschaftliche Feinderklärung zusammen mit Elmar Altvater verfasst (1976 in Heft 23). Die beiden Autoren setzten sich darin mit einer Schrift des Politologen Kurt Sontheimer auseinander, in der dieser das vorgebliche Elend linker Theorie beklagte - eine Kampfansage, die damals von ungeheurem publizistischen Getöse begleitet wurde. Daneben wurde nicht nur die SPD für Bodo Zeuner immer wieder zum Gegenstand der Analyse, sondern auch andere Parteien, so 1980 in Heft 38 (zusammen mit Hajo Funke) die CDU/CSU. Der konkrete Anlass dafür war die drohende Gefahr einer Kanzlerschaft von Franz-Josef Strauß bei der anstehenden Bundestagswahl. Von diesem war eine Politik nach dem Modell von Margret Thatcher in Großbritannien zu erwarten, und er betrieb seinen Wahlkampf mithilfe einer Politik des massiven Angstmachens und der gezielten Verunsicherung. Mit den Grünen, die 1983 in den Bundestag eingezogen waren, und ihrem Konzept der Basisdemokratie befasste sich Bodo Zeuner in ebendiesem Jahr in Heft 51. Er bezweifelte, dass das Konzept durchzuhalten war, da rätedemokratische Konzepte nicht ausreichend weiterentwickelt und erprobt worden waren, sodass die Partei letztlich doch die gängigen Politikformen des Repräsentativsystems übernehmen würde. Auf die »Parlamentisierung « der Grünen Partei kam Bodo Zeuner außerdem 1985 in Heft 61 noch einmal zurück und diagnostizierte, dass diese ihren eigenen Anspruch einer »Partei neuen Typs « kaum erfüllt hatte, in einem zunehmend schwierigen Verhältnis zu sozialen Bewegungen stand, aus denen sie hervorgegangen war, und sich mehr und mehr den Zwängen der Parteienkonkurrenz unterworfen sah. Im folgenden Jahr 1986 erschien in Heft 73 abermals ein Artikel zu den Gewerkschaften, diesmal zu ihrem Verhältnis gegenüber den linken Intellektuellen, für das Bodo Zeuner eine neue Qualität und Chancen zur verstärkten Zusammenarbeit sah. Beide Seiten hätten aus vergangenen Fehleinschätzungen dazugelernt. 
Alle diese Artikel sind von erheblichem partei- oder gewerkschaftshistorischen Interesse, berühren darüber hinaus aber auch Grundsatzfragen. Dies gilt ganz besonders für einen Beitrag, der 1995 unter dem Titel »Probleme des Klassenkampfs« im Betrieb in Heft 100 der PROKLA erschien, und auf den $2020 \mathrm{im}$ Editorial von Heft 200 (das den Problemen des Klassenkampfs - heute gewidmet war) noch einmal verwiesen wurde - mit gutem Grund. Denn Bodo Zeuner hatte bereits damals drei zentrale Punkte angesprochen, mit denen heutige Analysen von Klassenverhältnissen sich nach wie vor auseinanderzusetzen haben: Inwiefern die Gegenüberstellung von im Wesentlichen zwei Klassen für die Erklärung heutiger Strukturen kapitalistischer Gesellschaften ausreiche, wie es um das Verhältnis von Klassenlage und Klassenhandeln stünde, und ob die Zentralität der Klassenbegriffs zur Erklärung aller gesellschaftlichen Konflikte noch angemessen sei. Schließlich ist noch an einen Artikel von Bodo Zeuner aus dem Jahr 2007 zu erinnern, der als Gegenwartsdiagnose weiter Gültigkeit hat. Es handelt sich um seine Abschiedsvorlesung an der FU Berlin, die unter dem Titel Die freie Universität vor dem Börsengang in Heft 148 erschien. Darin beleuchtete er am Beispiel der eigenen Erfahrungen an der Universität die »Markt-Ökonomisierung der Wissenschaft «: die Kommodifizierung der Wissenschaftsprodukte, ihre Marktabhängigkeit und die "Unternehmisierung" des Universitätsbetriebs. Die PROKLA verdankt Bodo Zeuner wichtige Impulse. Stimmen wie seine fehlen.

Die PROKLA-Redaktion dankt Alexander Maschke, der in dieser Ausgabe als Gastredakteur mitgewirkt hat, für seine Ideen und sein Engagement.

\section{Der PROKLA-Förderverein}

Die PROKLA erscheint seit 1971 und bietet politisch engagierte sozialwissenschaftliche und ökonomische Analysen. Allein von den Verkaufserlösen kann sich die PROKLA nicht finanzieren, und in die Abhängigkeit von Parteien oder großen Verlagen wollte sie sich nie begeben. Deshalb wird die PROKLA von einem Förderverein herausgegeben, der »Vereinigung zur Kritik der politischen Ökonomie e.V.«, die jährlich in ihrer Vollversammlung die Redaktion der Zeitschrift wählt und die nächsten Themenschwerpunkte diskutiert.

Kritische Sozialwissenschaft kann nicht dem Markt überlassen werden. Ohne solidarische Strukturen und finanzielle Unterstützung sind Zeitschriften wie die PROKLA kaum möglich. Die finanziellen Beiträge der Vereinsmitglieder ermöglichen das Erscheinen der PROKLA, sie schaffen die Voraussetzungen für Kontinuität und Planbarkeit, wie sie für die Redaktionsarbeit unabdingbar sind. Wir freuen uns über weitere Mitglieder, regelmäßige Spenden oder einmalige Zuwendungen. Weitere Informationen teilen wir gerne per E-Mail mit (redaktion@prokla.de).

Vereinigung zur Kritik der politischen Ökonomie e.V. | Postbank Berlin | IBAN: DE17 100100100538135100 | BIC: PBNKDEFF 